\title{
Corrigendum
}

\section{Antidepressant use and colorectal cancer risk: a Danish population-based case-control study}

\author{
DP Cronin-Fenton, AH Riis, TL Lash, SO Dalton, S Friis, D Robertson and HT Sørensen
}

British Journal of Cancer (201I) 104, 1804. doi:10.1038/bjc.20II.176 www.bjcancer.com

(C) 201 I Cancer Research UK

Correction to: British Journal of Cancer (2011) 104, 188-192; doi:10.1038/sj.bjc.6605911

Upon publication in early 2011, the authors noticed a small error in the Methods section regarding the ATC codes for genotoxic and non-genotoxic TCA drugs, which may have caused some confusion for readers. The corrected text is presented below:

The list of ATC codes for TCA drugs should read:

'TCAs (genotoxic) (van Schaik and Graf, 1991, 1993): desipramine (N06AA01), imipramine (N06AA02), imipramine oxide (N06AA03), clomipramine (N06AA04), opipramol (N06AA05), trimipramine (N06AA06), doxepine (N06AA12), amoxapine (N06AA17), lofepramine (N06AA07); TCAs (non-genotoxic) (van Schaik and Graf, 1991, 1993): amitriptyline (N06AA09), nortriptyline (N06AA10), protriptyline (N06AA11), dosulepine (N06AA16)'. 